\title{
e-Migrinter
}

17 | 2018

Enseigner les migrations internationales

\section{Les migrations internationales dans l'enseignement belge francophone}

Dynamiques d'une reconnaissance dans la pensée et les savoirs hégémoniques

\section{Nouria Ouali}

\section{(2) OpenEdition}

\section{Journals}

Édition électronique

URL : https://journals.openedition.org/e-migrinter/1063

DOI : 10.4000/e-migrinter.1063

ISSN : 1961-9685

Éditeur

UMR 7301 - Migrinter

Référence électronique

Nouria Ouali, «Les migrations internationales dans l'enseignement belge francophone », e-Migrinter [En ligne], 17 | 2018, mis en ligne le, consulté le 20 mai 2021. URL : http://journals.openedition.org/emigrinter/1063 ; DOI : https://doi.org/10.4000/e-migrinter.1063

Ce document a été généré automatiquement le 20 mai 2021.

Tous droits réservés 


\section{Les migrations internationales dans l'enseignement belge francophone}

Dynamiques d'une reconnaissance dans la pensée et les savoirs hégémoniques

Nouria Ouali

1 L'histoire migratoire de la Belgique remonte au XVIe siècle quand de nombreux Belges émigrent vers l'étranger (Europe et l'Amérique du Nord) pour des raisons économiques ou politiques, et ce jusqu'à la première guerre mondiale. Au cours du XXe siècle, les flux s'inversent et la Belgique accueille différentes migrations d'asile et de travail originaires des pays du Sud et de l'Est de l'Europe, d'Afrique du Nord, de l'exYougoslavie et de la Turquie $^{1}$. Cependant, comme d'autres pays d'anciennes immigrations en Europe, l'État et ses institutions connaissent quelques difficultés à valoriser cette histoire tant à l'égard de ses émigrants que de ses immigrés.

Depuis quelques années, les savoirs universitaires sur les migrations internationales, notamment en histoire et en sociologie, et les expertises sur ces questions ont abondés grâce à la création de centres de recherches spécialisés sur cet objet. Pourtant, ces nombreuses connaissances disponibles peinent à se diffuser et à trouver une place à part entière parmi les matières « valables » à enseigner, alors que les migrants et leurs descendants constituent une composante non négligeable de la société ${ }^{2}$. Comment saisir ce paradoxe et les obstacles persistants, dans le système éducatif, à reconnaître ce fait sociologique majeur qui a profondément transformé la société?

3 La réponse se trouve en partie dans l'incapacité des acteurs de l'éducation à penser la présence définitive des migrants et leurs descendants dans la société belge et, par voie de conséquence, de l'inscrire dans les enseignements tel que, par exemple, le cours d'histoire. I

4 L'analyse repose sur l'examen, d'une part, des documents du Service général des Affaires pédagogiques, de la Recherche en Pédagogie et du Pilotage de l'Enseignement de la Communauté française qui valide les programmes imposés aux quatre types d'enseignement du secondaire ${ }^{3}$ depuis 2001-2002: aux 2e et 3e degrés des cours d'histoire, de géographie, de morale laïque et de sciences sociales de l'enseignement 
général et technique de transition, et l'enseignement professionnel et technique de qualification. D'autre part, les cours et formations affichés sur les sites internet de quatre universités francophones: l'Université Libre de Bruxelles, l'Université Catholique de Louvain (autonomes subsidiées) et les universités de Liège et de Mons (universités publiques de la Communauté française).

\section{Une revendication récursive}

5 Les premiers ouvrages qui rendent justice à l'histoire des migrations ont été publiés il y a à peine vingt-cinq ans. Ils ont, d'ailleurs, relancé le débat sur l'enseignement des migrations internationales dans les programmes scolaires. Portée au départ par le mouvement associatif mobilisé pour l'égalité des droits, le droit de vote des étrangers et contre le racisme, la discussion se focalise sur la création d'un cours d'histoire des immigrations. Celui-ci est présenté à la fois comme une condition de l'intégration dans la société des migrants et leurs enfants et comme un moyen de lutter contre le racisme.

Dans un article paru en 1988 dans les Cahiers de Clio, l'historienne Anne Morelli ${ }^{4}$ plaidait pour la création d'un cours spécifique sur l'histoire de l'immigration à l'école secondaire; en 2004, elle exprimait sa "désespérance face à la lenteur des changements sur le plan pédagogique » $(2004: 13)$ et en 2011 , elle regrettait toujours l'impasse dans laquelle se trouvait cette discussion.

7 Pourtant, depuis le début des années 2000 , des voix s'élèvent pour revendiquer des commémorations, des symboles et des lieux afin d'inscrire l'immigration dans le paysage et dans le récit historique de la Belgique. Pour combattre l'oubli et le dénigrement, des migrants et leurs descendants cherchent à valoriser leurs expériences, leurs rôles dans la société souvent dénigrés, et à forger, plus globalement, une image positive de l'immigration. Ainsi, depuis 2002, le site minier du Bois du Cazier commémore l'incendie du 8 août 1956 en Wallonie qui a tué 262 travailleurs dont 167 travailleurs immigrés (parmi lesquels 136 italiens) et se transforme en musée de l'industrie. Le cimetière de Chastre où sont enterrés des tirailleurs Marocains tombés lors de la bataille du 8 mai 1940 fait, depuis 15 ans, l'objet d'une cérémonie annuelle d'hommage à ces soldats oubliés de l'histoire de la résistance au nazisme. En 2003, des descendants de migrants marocains créent l'Espace mémorial de l'immigration marocaine (EMIM) pour constituer les archives de cette immigration et célèbre leur histoire, en 2004, autour du 40e anniversaire de la signature de la convention bilatérale de main d'œuvre; les Turcs et les Italiens feront de même en 2006 et 2014. Plus récemment, des associations de migrants d'origine subsaharienne se mobilisent autour de la question coloniale et revendiquent l'enseignement de la colonisation et de l'immigration africaine dans les écoles 5 .

8 Ces demandes émergent au moment où le système éducatif est fortement disqualifié et fait l'objet de coupes budgétaires importantes qui se traduisent par l'abandon de la plus importante réforme pédagogique démocratique (l'enseignement rénové6) au profit d'une politique de discrimination positive mise en œuvre au milieu des années 1980 (Beckers, 1998). Ces mesures ciblent désormais des territoires et des établissements qui accueillent notamment un nombre significatif d'enfants de migrants et issus des classes populaires. Comme ailleurs, cette politique renforce la stigmatisation des établissements et des enfants qui les fréquentent, et accélère le processus de 
ségrégation sociale et ethnique des écoles déjà fortement exacerbé par la logique de quasi-marché propre au système scolaire belge $\mathrm{e}^{7}$.

Dans ce contexte, la présence des enfants d'immigrés est associée à la baisse des niveaux de performances scolaires et à des éléments perturbateurs du bon fonctionnement de l'école (échec, absentéisme, décrochage, incivilités et violences scolaires). L'irruption des questions identitaires et de l'islam à l'école (cours de religion et foulard islamique) dans les années 80 et 90 a favorisé le développement d'un discours dominant qui incrimine l'origine culturelle, la religion musulmane et les " problèmes » identitaires de ces élèves dans la production de leurs difficultés scolaires (Ouali, 2007 ; 2006).

10 L'institution scolaire qui a historiquement joué un rôle non négligeable dans l'intégration et la promotion sociale des classes populaires, se révèle depuis les années 1980 de plus en plus inégalitaire et particulièrement sélective à l'égard des enfants de migrants. L'inégalité se manifeste, d'un côté, à travers un échec scolaire plus fréquent et une pratique hors norme de l'orientation scolaire vers les enseignements professionnel et spécial ${ }^{8}$ qui sera, finalement, officiellement reconnue abusive et discriminatoire en 1999. De l'autre côté, la liberté constitutionnelle de choix de l'école des parents migrants est régulièrement violée à travers les refus d'inscription de leurs enfants sur la base soit des résultats scolaires, des comportements ou sans raison valable', soit en conséquence de l'interdiction du voile islamique imposé dans la quasitotalité des écoles sur base d'une loi générale (Flandre) ou d'un règlement intérieur des établissements (Bruxelles et Wallonie).

\section{Le système éducatif et les enfants de migrants}

11 En vérité, les acteurs du système éducatif ont peu pensé la question migratoire et sa prise en compte dans le cursus scolaire. Ainsi, malgré les recommandations de chercheurs en éducation, formulée dès les années 1960 (De Coster \& Derume, 1962), visant à soutenir la scolarité des enfants d'immigrés les autorités éducatives ont tardé à agir puis se sont limitées à des mesures ponctuelles et expérimentales. Cette attitude repose sur une double perception des enfants de migrants : une présence temporaire qui oriente les contenus et les approches pédagogiques vers leur préparation au retour au pays d'origine ${ }^{10}$ et la naturalisation de leur échec scolaire en raison de leur langue et de leur culture d'origine.

12 Cette présence temporaire fut envisagée jusqu'en 1989 où le gouvernement fédéral propose une politique d'intégration des migrants qui entraîne un renouveau de la stratégie éducative et un changement de la perception des enfants de migrants. D'abord, l'échec scolaire de ces enfants n'est plus essentiellement attribué à leur langue et à leur culture d'origine, mais à l'attitude "démissionnaire " des parents et aux comportements inappropriés des jeunes (violence, absentéisme et décrochage scolaire). Ensuite, la politique éducative se territorialise et la nationalité ou l'origine étrangères des élèves constitue un des critères parmi d'autres ${ }^{11}$, de financement des écoles à "discrimination positive " ou à "encadrement différencié » (Ouali 2007). Enfin, les écoles normales enseignent désormais les théories interculturelles et définissent l'approche interculturelle comme nouvelle compétence à acquérir aux futurs enseignants. En pratique, les cours portent essentiellement sur des techniques (pédagogie, communication, etc.) qui visent, selon les autorités éducatives, à mieux 
"gérer" les populations scolaires d'origine étrangère. Au final, les migrations continuent d'être un point aveugle de ces enseignements.

Soulignons que dans les années 80 , les autorités éducatives, contraintes et forcées par la directive européenne, organisaient des expériences pilotes d'éducation interculturelle dans quelques écoles francophones qui usaient toutes des mêmes recettes: l'apprentissage de la langue d'enseignement (français ou néerlandais), les cours de langues et de cultures d'origine (LCO) organisés avec le concours des pays d'origine et la formation à la pédagogie interculturelle des enseignants. Il n'est pas question ici d'enseigner l'histoire ou la sociologie des migrations alors qu'elles auraient dû y trouver leur place, si l'on s'en réfère à l'esprit du Conseil de l'Europe (Crispin \& Keith 1989) qui inventa, en 1976, le concept d'éducation interculturelle ${ }^{12}$.

14 L'incapacité de ces expériences à traiter de l'échec et les inégalités scolaires - qui seront qualifiées de "pédagogie de loisir» (CRPI, 1990) - entrainera de nombreuses critiques notamment sur leur approche très culturaliste ${ }^{13}$ et justifiera l'abandon de ces pratiques. $\mathrm{Au}$ début des années 2000, le rapport entre les descendants de l'immigration et leur scolarisation est à nouveau redéfini dans un contexte marqué par l'émergence de l'islam dans l'espace scolaire. Ainsi, en 2005, la Commission du Dialogue Interculturel (CDI) ${ }^{14}$ auditionne des acteurs scolaires qui, tout en reconnaissant les problèmes de discrimination et de ségrégation scolaires, reformulent leur perception des problèmes scolaires des enfants de migrants essentiellement en lien avec le port du foulard islamique, le prosélytisme religieux, l'homophobie, le sexisme à l'égard des enseignantes, le rejet du contenu de l'enseignement (libre examen, darwinisme, éducation sexuelle), le refus de participer aux activités sportives, et les pressions exercées sur les musulmanes non voilées. Partant toujours d'une conception "déficitaire " (c'est nous qui soulignons) de ces élèves, les propositions formulées consistent alors à combler leurs « carences » (c'est nous qui soulignons) notamment en matière d'histoire de la démocratie, de l'émancipation, de la liberté et de l'égalité entre les hommes et les femmes, et d'instruction civique et citoyenne. La Commission suggère deux pistes d'action: la première propose l'intégration de "la réalité des trajets migratoires ou de l'exil, l'apport des cultures non européennes à la connaissance, aux idées et aux découvertes » dans les cours d'histoire, de géographie, de littérature et des sciences (Commission, 2005 : 89). La seconde invite les autorités politiques à créer un musée national de l'immigration ${ }^{15}$ censé mettre en valeur les parcours migratoires et l'histoire des pays et des régions d'origine ; un musée qui collaborerait avec les écoles et les chercheurs. Ces pistes n'ont pas réellement vu le jour et le projet de musée a été abandonné comme celui envisagé à Bruxelles ${ }^{16}$.

Le rapport du système éducatif belge aux migrants et leurs enfants révèle le même paradoxe que Sayad souligne pour la France :

"L'immigré et sa famille sont définis (hier comme aujourd'hui) par le provisoire alors que l'institution scolaire a partie liée à la durée, sa vocation fondamentale consistant à prendre possession de l'avenir. " (Cité et souligné par Falaize \& Laacher, $2014: 229$ ).

Ce provisoire contribue ainsi à renforcer l'extériorité des immigrés et de leurs enfants à la société " pour penser socialement les logiques de scolarisation " (Falaize \& Laacher, 2014 : 234). L'extériorité des générations successives en Belgique se marque à travers la catégorie "allochtone $»^{17}$ utilisée pour désigner les personnes possédant ou non la nationalité belge dont au moins un des parents ou des grands-parents est né en dehors 
de la Belgique. Il s'agit plus particulièrement des personnes issues de l'immigration non-européenne.

\section{Les migrations dans l'enseignement secondaire}

La réforme éducative de 1997 (décret « Missions ») apporte quelques modifications des programmes et inclut, depuis l'année 2001-2002, la thématique migratoire aux 2e et 3e degrés de l'enseignement secondaire général, technique et professionnel. Elle est désormais inscrite au programme de quatre cours: histoire, géographie, sciences sociales et morale laïque ${ }^{18}$ et fait l'objet soit d'unités ${ }^{19}$ des cours d'histoire et de géographie généralement enseignés à un grand nombre d'élèves, soit d'un module (plusieurs unités) de l'option de base ${ }^{20}$ en sciences sociales suivi par un faible nombre d'étudiants. L'option de base en histoire ne fournit aucuns détails sur le contenu du programme et l'option en géographie ne mentionne pas cette matière. Le cours de morale laïque, qui est à option, aborde indirectement la migration par le biais de la thématique liée à la citoyenneté et à l'intégration.

17 Les programmes privilégient tous les questions sur la citoyenneté active, la diversité, l'environnement et l'insertion professionnelle. Ces matières doivent permettre aux jeunes de "comprendre le monde pour être un citoyen responsable». L'analyse du contenu des cours montre que l'approche de la question migratoire est très superficielle dans sa dimension historique et que les thèmes abordés sont très ciblés : stéréotypes, mariages forcés, « problèmes scolaires » des enfants d'immigrés, logement, délinquance et réfugiés. Examinons à présent l'objet de chaque matière et le temps consacré à la migration.

\section{Le cours d'histoire}

Ce cours est dispensé aux élèves de l'enseignement général, technique et professionnel et fait partie des cours généraux obligatoires enseignés à tous les élèves des trois degrés d'enseignement (voir note 5). Cependant, seuls les programmes des cours des $2 \mathrm{e}$ et $3 \mathrm{e}$ degrés ${ }^{21}$ abordent la thématique migratoire. Le nombre d'heures de cours varie entre 3 et 4 périodes (de 50 minutes) par semaine pour les cours obligatoires en fonction du réseau d'enseignement ou de l'option de base (4 périodes).

Le programme de l'enseignement professionnel et technique du 2e degré (donc 3e et 4e années) aborde la migration dans le module intitulé « identités et migrations » et fait l'objet de trois unités d'enseignement distinctes $(5,6$ et 9): elle est abordée à la fois sous l'angle des migrations des Belges aux XIXe et XXe siècles et des migrations étrangères en Belgique au XXe siècle.

L'unité 5 est consacrée aux mouvements migratoires internes et vers l'étranger des Belges. Il s'agit en l'occurrence des migrations économiques des ouvriers flamands qui ont quitté massivement la Flandre (sujette aux crises agricoles et climatiques, et aux famines au XIXe siècle) vers la Wallonie industrialisée qui était à l'époque la $2 \mathrm{e}$ puissance industrielle au monde. Le cours présente le profil, les origines de cette émigration, son ampleur et l'intégration des Flamands (identité, langue, religion, modes de vie) en Wallonie. 
21 L'unité 6 porte sur La colonisation, la décolonisation : les relations entre colons et colonisés au Congo belge (1885-1960). Elle examine, selon la formule du programme, "l'émigration volontaire » des Belges pendant la période coloniale organisée par l'État entre 1885 et 1960. La matière met moins l'accent sur la migration (trajectoires, profils, régions, missions, etc.) que sur le processus colonial, son contexte et son objectif explicite d'exploitation des richesses naturelles.

22 L'unité 9 aborde Les migrations internationales économiques et politiques vers la Belgique aux XXe et XXIe siècles. Elle distingue les migrations de travail et familiales organisées par l'État et analyse les causes des migrations, les politiques migratoires, les profils, leur ampleur, leur localisation dans les régions du pays et l'intégration des immigrés (identité, langue, religion, modes de vie) en lien avec le développement économique.

23 Le programme de l'enseignement général au 3e degré (en 5e année) traite la migration dans le cadre des phénomènes démographiques de quelques pays européens et aux États-Unis : évolution de la population globale, taux de natalité, mortalité, etc. Le concept de «migration» est décliné sous ses multiples facettes: émigration, immigration, migration interne, externe, exode rural, migration économique, politique, fiscale, etc. L'unité consacrée à l'Union européenne examine la politique migratoire de l'UE et l'Espace Schengen qui contrôle et limite la mobilité des étrangers. L'option de base d'histoire (4h) dans le 3e degré ne mentionne rien sur le contenu des unités d'apprentissage.

\section{Cours de géographie}

Comme le cours d'histoire, la géographie est enseignée aux élèves des trois degrés de l'enseignement secondaire. Le nombre d'heures enseignées varie entre 1 et 4 périodes (pour l'option de base) par semaine. Les programmes distinguent l'enseignement général et technique de transition (vers l'enseignement supérieur) de l'enseignement professionnel et technique de qualification (vers un métier) ${ }^{22}$.

25 Le programme de l'enseignement général et Technique (de transition) du cours de géographie des 2è et 3è degrés comprend plusieurs unités d'enseignement sur la migration, ce concept étant considéré comme une des notions fondamentales du cours. Les migrations sont analysées comme un facteur décisif de transformations des espaces et des sociétés. Au 2e degré, la migration est abordée dans le chapitre sur les Etats-Unis et la Chine où les politiques d'immigration, l'histoire migratoire (migrations européennes, migrations forcées d'esclaves, hispaniques et asiatiques), les politiques d'intégration sont examinées au regard des réalités économiques et du développement démographique. Au 3e degré, les dynamiques migratoires dans l'Union européenne sont développées et distinguées selon les zones d'immigration et d'émigration et le rôle des migrations dans le développement $\mathrm{du}$ système socio-économique et dans la configuration des espaces. Le programme de l'option de base de géographie de 4 périodes de cours ne prévoit aucune matière sur les migrations.

Le programme de l'enseignement professionnel et technique (de qualification) propose au 2e degré (4e année) deux unités d'apprentissage consacrées aux migrations dans le module intitulé «Les migrations en Belgique de l'échelle locale à l'échelle mondiale». Ces unités mettent l'accent sur plusieurs dimensions: les concepts (immigration, émigration, flux, zone d'appel, etc.), l'origine des flux sous l'angle des réservoirs migratoires, l'importance des flux et les destinations (composante du 
territoire), l'espace (pôles migratoires Europe et États-Unis, les limites politiques de l'UE et de la Belgique, les villes belges et mondiales), les liens entre le déplacement des populations et les conditions de vie et les identités des migrants, et la diversité socioculturelle.

\section{L'option de base en sciences sociales}

27 L'enseignement secondaire général offre aux élèves au 3e degré différentes options de base notamment en sciences sociales ${ }^{23}$. Cette option approfondit les notions de base de l'anthropologie sociale, la démographie, l'économie, du droit, du politique et de la sociologie. L'objectif n'est pas de former des techniciens des sciences sociales mais de permettre aux élèves de prendre conscience et d'analyser certains phénomènes sociaux.

Le programme dans le 2e degré est, sans conteste, celui où la thématique migratoire est la plus élaborée. En 3e année, les élèves découvrent la diversité des sciences sociales et deux thématiques imposées: la famille et l'immigration en Belgique. En 4e année, le cours est davantage axé sur les cultures premières (encore dénommée "archaïque " dans le document de l'éducation) ou issues d'autres régions du monde.

Le programme de la 3e année propose de mieux appréhender l'immigration et de la traiter en rapport avec l'actualité. Les enseignants font donc usage de documents spécialisés, d'articles de presse et d'émissions ou journaux télévisés. Le cours est organisé en quatre partie : la première est consacrée à l'histoire de l'immigration en Belgique décomposée en trois périodes (avant 1914, après 1920 et après la Deuxième Guerre mondiale). La deuxième partie présente la situation statistique de l'immigration et analyse l'évolution de la population étrangère et le profil des migrants (pyramide des âges, régions d'origine et d'installation, quelles activités exercent-ils et dans quels secteurs, les taux de chômage etc.). La troisième partie analyse l'immigration en lien avec la sécurité sociale pour déconstruire les stéréotypes associés aux migrants (par exemple «paresseux et profiteurs de la sécurité sociale »). La quatrième partie aborde la sociologie de l'immigration à travers la scolarité, le logement, la délinquance et les mariages. Le cours examine les concepts (émigré, immigrant, étranger, réfugié, clandestin etc.), les idées reçues et les ressorts de la peur de l'étranger. Il aborde également les questions relatives à l'intégration, au racisme et à l'extrême droite.

\section{Le cours de morale laïque}

Dans l'enseignement public, ce cours de morale est laissé au choix des parents ou de l'élève (s'il est majeur) avec les cours de religion ${ }^{24}$, il n'est pas organisé dans les écoles de l'enseignement confessionnel subventionné. Il est conçu pour les élèves qui ne se réclament d'aucune confession et souhaitent

« résoudre leurs problèmes moraux sans se référer à une puissance transcendante ni à un fondement absolu, par le moyen d'une méthode de réflexion basée sur le principe du libre examen. $»^{25}$

31 Seul le programme de 3e année (2e degré) aborde la thématique de la migration dans le module consacré à "L'humanité et les droits de l'Homme », dans la section relative à la citoyenneté démocratique où la question des immigrés et des réfugiés est très sommairement abordée. Elle est enseignée en lien avec l'apprentissage de certaines 
valeurs et des questions de société (tolérance, dignité, identité, différence, égalité, humanité, droit). Le cours examine une série de préjugés associés aux immigrés (il mentionne "mangent-ils notre pain ou nous aident-ils à le cuire?»), à la condition faite aux réfugiés (le thème sur "les barbelés avant l'expulsion!»), à l'égalité et au droit à la différence. Une partie du cours est consacrée au racisme dans le contexte de l'histoire des Noirs aux USA.

L'analyse des programmes de l'enseignement secondaire confirme que les autorités éducatives consacrent peu d'heures de cours à la thématique migratoire, excepté dans l'option de base en sciences sociales. Les contenus adoptent une approche mainstream des populations migrantes et des "problèmes sociaux» qu'elles posent à la société belge en les traitant sous l'angle de l'intégration, l'identité culturelle, la religion ou l'égalité entre les femmes et les hommes. L'absence de déconstruction de ces questions éminemment sensibles et complexes peut, malgré les bonnes intentions et les bons sentiments des enseignants (Sayad, 2014), contribuer à renforcer la stigmatisation de certains groupes, en particulier ceux identifiés comme musulmans réels ou supposés. Aucun cours n'examine sérieusement la migration internationale dans ses différentes facettes (émigration/immigration), les disciplines multiples (histoire, géographie, sociologie, économique, politique) et sous un angle critique. Ces constats renvoient inévitablement à la formation de base des enseignants. Nous verrons, dans la section suivante, que les formations existantes ne ciblent pas suffisamment ces professionnels et que l'approche des formations pédagogiques trop souvent axées sur l'identitaire et le culturel, n'offrent pas les outils nécessaires pour enseigner une des questions les plus complexes de nos sociétés contemporaines.

\section{Les formations universitaires}

Les enseignements sur les migrations internationales à l'université sont relativement récents, peu nombreux et d'une toute autre ampleur que ceux organisés dans le secondaire. Depuis quelques années, des modules, certificat et finalité spécialisée en sociologie ont été créés, généralement par des chercheurs spécialisés sur ces questions.

Les résultats présentés ici ne sont pas exhaustifs, ils portent sur l'analyse des cours essentiellement organisés au niveau des masters des cursus de base et les formations pédagogiques ${ }^{26}$ de quatre universités francophones (Bruxelles, Mons, Louvain et Liège), et la formation continue d'une seule université (voir plus loin le tableau récapitulatif). La recherche s'est effectuée sur l'intitulé comprenant au moins un des termes suivants : " migration, immigration, étrangers ou réfugiés, diversité, interculturel, intégration, multiculturalisme, ethnicité, racisme». La migration n'est pas envisagée au sens restreint de mobilité internationale, mais au sens large incluant toute expérience en lien avec la migration. Les cours ont été analysés sur base des contenus présentés sur les sites internet des universités, l'accès aux supports des cours comme les power points ou les syllabus n'a pas été possible.

Les enseignements identifiés sont dispensés dans les différentes disciplines des sciences sociales telles que la géographie, l'histoire, la démographie, la politique, le droit et la sociologie. Ils abordent les migrations internationales sous des angles et des échelles variées (nationale, régionale, européenne ou internationale). Les formations en communication, histoire, ou psychologie mettent davantage l'accent sur le contact des cultures, les relations ou la psychologie interculturelles et intergroupes. Les 
enseignements s'inscrivent soit dans les cursus universitaires classiques des étudiants de master, soit dans la formation continue destinée aux professionnels du privé et du public qui représentent une source de financement nouvelle et un vecteur de recrutement des futurs étudiants ${ }^{27}$.

Ainsi, la politique de promotion de la diversité mise en place dans les secteurs des entreprises publiques et privées depuis 2007 a créé des besoins importants en formation, notamment dans les départements du personnel qui abordent la diversité sous l'angle managérial.

La Belgique francophone ne dispose pas de formation telle que le Master national de l'Université de Venise (Master sull'immigrazione) qui forme tous les étudiants du pays. Les enseignements identifiés se présentent en majorité sous une forme éclatée car probablement créées au gré des intérêts personnels d'enseignants, des opportunités financières locales ou des demandes régionales récentes des pouvoirs publics ou des acteurs privés. Ainsi, les formations analysées se présentent sous quatre formes: une activité d'apprentissage (chapitre d'un cours), des unités d'enseignement ${ }^{28}$ incluses dans un module ou des formations généralistes; une d'une finalité spécialisée dans le cadre d'un master; et une formation certificative et modulaire ouverte aux professionnels du social, des entreprises privées et du secteur public en charge des populations migrantes. Examinons le contenu de ces quatre formes d'enseignement identifiées.

\section{Une activité d'apprentissage}

L'activité intitulée «Les migrations internationales et ses relations avec la mondialisation » de l'unité d'enseignement Démographie et pays en voie de développement est présenté ici comme un exemple de cours ${ }^{29}$ de $24 \mathrm{~h}$ en démographie qui consacre $2 \mathrm{~h}$ à $4 \mathrm{~h}$ aux migrations. Dans cette même discipline de démographie, une autre université a plutôt fait le choix de créer une unité d'enseignement et de lui dédier 30h destinées aux seuls étudiants de master (voir infra).

Le chapitre définit le concept de "migrations internationales» et ses méthodes d'estimation, la croissance des flux dans le monde et en Belgique, la géopolitique des flux migratoires, les trajectoires et les circuits de la migration internationale, les théories sur les migrations et la question des transferts financiers. Le cours est destiné aux bacheliers et aux masters: il est obligatoire pour les bacheliers en sciences géographiques et en sciences humaines et sociales, et pour les étudiants du master en sciences de la population et du développement. Il est optionnel pour les bacheliers en sciences politiques, en sociologie et anthropologie, pour les finalités « didactiques » et « développement territorial » des masters en sciences géographiques et pour le master en sciences actuarielles.

\section{Les unités d'enseignement}

40 C'est sous cette forme que nous avons identifié le plus d'enseignements sur les migrations dans les masters des quatre universités. Seul un cours est dispensé aux bacheliers. Le plus souvent ces cours sont optionnels, valent entre 3 et 6 crédits avec un nombre d'heures de cours variant entre $24 \mathrm{~h}$ (le plus fréquent) et $36 \mathrm{~h}$ maximum. La plupart de ces enseignements sont prodigués en français, mais quelques-uns le sont en 
anglais (accessibles aux étudiants d'une autre université européenne) et un seul cours est libellé en néerlandais mais ne présente aucun contenu.

Le nombre de cours varie selon les universités : Mons en propose 1 seul, Bruxelles 5, Louvain-La-Neuve 5 et Liège 12. Ils couvrent plus ou moins les mêmes disciplines: sociologie, anthropologie, droit, sciences politiques, géographie et démographie. Étonnement, on observe que l'histoire y est absente. L'intitulé des cours, la discipline, le nombre de crédits consacrés à la thématique migratoire et l'université qui l'organise sont repris dans le tableau ci-dessous.

L'organisation des cours montre qu'ils sont souvent mutualisés par différents masters et/ou finalités. Le contenu, tel que présenté dans les fiches de cours disponibles sur les sites universitaires, révèle la dominance de quatre disciplines: sociologie et anthropologie, sciences politiques, démographie et droit.

Tableau $n^{\circ} 1$ : Intitulés, disciplines et nombre de crédits des cours sur les migrations, dispensés dans quatre universités francophones de Belgique

\begin{tabular}{|c|c|c|}
\hline Intitulé des cours & Disciplines & $\begin{array}{l}\text { Nombre } \\
\text { d'heures }\end{array}$ \\
\hline \multicolumn{3}{|l|}{ Université Libre de Bruxelles } \\
\hline Sociologie des migrations & Sociologie & $24 \mathrm{~h} / 5$ crédits \\
\hline Migrant Integration Policies in Europe & Sociologie & $36 \mathrm{~h} / 5$ crédits \\
\hline $\begin{array}{l}\text { Migratie en de multiculturele samenleving (Migration et la } \\
\text { société multiculturelle) }\end{array}$ & $\begin{array}{l}\text { Sciences } \\
\text { géographiques }\end{array}$ & 6 crédits \\
\hline European Asylum and Migration Law & Droit & $24 \mathrm{~h} / 5$ crédits \\
\hline Droit belge des étrangers & Droit & $24 \mathrm{~h} / 5$ crédits \\
\hline $\begin{array}{l}\text { Les migrations internationales et ses relations à la } \\
\text { mondialisation }\end{array}$ & Démographie & Chapitre \\
\hline \multicolumn{3}{|l|}{ Université de Mons } \\
\hline Socio-anthropologie des migrations & Anthropologie & $30 \mathrm{~h} / 5$ crédits \\
\hline \multicolumn{3}{|l|}{ Université Catholique de Louvain } \\
\hline Relations interculturelles et migrations & Sociologie & $30 \mathrm{~h} / 3$ crédits \\
\hline Sociologie des migrations & Sociologie & $30 \mathrm{~h} / 4$ crédits \\
\hline Anthropologie des migrations & Anthropologie & $\begin{array}{l}22,5 \mathrm{~h} / 5 \\
\text { crédits }\end{array}$ \\
\hline $\begin{array}{l}\text { Urbanisation, migrations internes et migrations } \\
\text { internationales }\end{array}$ & Démographie & $30 \mathrm{~h} / 5$ crédits \\
\hline
\end{tabular}




\begin{tabular}{|c|c|c|}
\hline Droit et migrations & Droit & $30 \mathrm{~h} / 5$ crédits \\
\hline \multicolumn{3}{|l|}{ Université de Liège } \\
\hline Migrations, citoyenneté et dynamiques interculturelles & Science politique & $30 \mathrm{~h} / 6$ crédits \\
\hline Sociology of Migration & Sociologie & $30 \mathrm{~h} / 6$ crédits \\
\hline Migrations, citoyenneté et dynamiques interculturelles & Science politique & $30 \mathrm{~h} / 6$ crédits \\
\hline Theories of integration, diversity and multiculturalism & Science politique & $30 \mathrm{~h} / 6$ crédits \\
\hline Migration Transnationalism and Development & Science politique & $30 \mathrm{~h} / 6$ crédits \\
\hline Current Debates in Migration and Ethnic Studies & Sociologie & $30 \mathrm{~h} / 6$ crédits \\
\hline $\begin{array}{l}\text { Migration Policies and Multiculturalism in the European } \\
\text { Union }\end{array}$ & Science politique & $30 \mathrm{~h} / 6$ crédits \\
\hline Arts. Ethnicity and Migration & Sociologie & $30 \mathrm{~h} / 6$ crédits \\
\hline $\begin{array}{l}\text { Undocumented Migration, social movements and public } \\
\text { policy }\end{array}$ & Science politique & $30 \mathrm{~h} / 6$ crédits \\
\hline Using quantitative methods in Migration Studies & Sociologie & $30 \mathrm{~h} / 6$ crédits \\
\hline Citizenship and Political Participation of Minorities & Science politique & $30 \mathrm{~h} / 6$ crédits \\
\hline Research methods in Migration Studies & Sociologie & Non précisé \\
\hline
\end{tabular}

Les cours de sociologie et d'anthropologie adoptent le plus souvent une approche macro mais certains cours ont des accents plus micro en pointant les stratégies migratoires et identitaires, les relations de parenté ou les parcours d'exclusion et de précarité des migrants. Curieusement, dans l'approche macro peu de cours abordent la dimension historique et un seul cours d'anthropologie traite de la question des mémoires et de la transmission. Les théories sur les migrations, l'ethnicité, l'intégration, le multiculturalisme, la diversité ou les relations interculturelles sont les thèmes les plus communément traités. Parmi les cours orientés sciences politiques, les politiques migratoires, d'intégration, de participation et de citoyenneté tiennent le haut du pavé ; un cours d'anthropologie examine la question de la violence de l'État à l'égard des migrants (candidats réfugiés, sans papiers) dans le cadre de la fermeture des frontières. En démographie, l'unité d'enseignement décrit les migrations internes et internationales sous l'angle de leurs impacts sur les processus d'urbanisation: développement urbain, exode rural, la périurbanisation et les migrations de retour. Le cours renvoie également aux théories sur les migrations internationales, aux profils, causes et impacts démographiques notamment sur l'environnement. Enfin les cours de droit étudient la condition des migrants à partir de différentes branches du droit et aux échelles nationale, européenne et internationale. Ainsi, les enseignements abordent les droits humains, le droit administratif et de séjour des réfugiés et des étrangers, le droit pénal (extradition, répression transfrontière, terrorisme), le droit 
civil (famille, enfants, héritage), le droit international privé (statut personnel et conflits de lois) et le droit européen relatif à la libre circulation des citoyens de l'UE.

\section{La finalité spécialisée d'un master}

44 Une seule université (Liège) propose une finalité en Immigration Studies qui s'inscrit dans l'offre des cursus proposés aux étudiants du master en sociologie. Cette formation est ouverte aux étudiants de l'Universitad Pompeu Fabra (UPF) de Barcelone qui organise un master en Immigration and management. Ces étudiants bénéficient ainsi d'une double diplomation.

La plupart des cours sont dispensés en anglais, la maîtrise de cette langue est un des critères de sélection (sur dossier). La finalité vise à former différents professionnels locaux (experts, chargés de projets, chercheurs) qui traitent les questions migratoires pour les autorités gouvernementales et locales et les institutions universitaires, mais également des experts au service des organisations internationales (l'Organisation internationale pour les Migrations (OIM), l'Agence des Nations Unies pour les réfugiés (UNHCR) ou encore l'Organisation Internationale du Travail (OIT)), des agences de développement, des think-tanks et ONG, de la presse et des représentations diplomatiques.

La formation aborde les aspects politiques, sociologiques, juridiques et économiques des migrations dans une perspective multidisciplinaire et reprend, grosso modo, les thèmes abordés dans les unités d'enseignement analysés précédemment. L'originalité de cette finalité réside dans l'offre de thématiques neuves (l'art et la migration et les méthodologies appliquées aux migrations) et sur l'enseignement en anglais. Par ailleurs, elle dispense les cours de base des sciences sociales (épistémologie des sciences sociales, sociologie de la critique sociale, du contrôle social, globalisation et société civile, sociologie de l'action publique ou les politiques éducatives, l'éducation aux médias) qui proposent des outils et une dimension critiques essentiels. Elle élargit également ses thématiques à la géopolitique de la Méditerranée occidentale et à l'anthropologie des cultures arabo-islamiques.

\section{Le certificat interuniversitaire spécialisé}

47 Ce certificat en " Migration, diversité ethnique et relations interculturelles » est par des centres de recherche sur les migrations internationales de deux universités francophones (Bruxelles et Liège). Les enseignants appartiennent essentiellement au Groupe d'études sur les relations ethniques, les migrations et l'égalité et au Centre d'études de l'ethnicité et des migrations qui ont produit une expertise reconnue depuis des années.

Le certificat est organisé dans le cadre de la formation continue pour les salariés (les employés et cadres des secteurs privé et public, le personnel du secteur non marchand, GRH, syndicats) qui peuvent bénéficier d'un congé éducation ${ }^{30}$. Il vise en particulier les professionnels du social et de l'entreprise afin

«d'acquérir de nouvelles compétences et de devenir des acteurs de changement facilitant l'implémentation d'une bonne gestion de la diversité culturelle au sein de votre entreprise ». 
La formation cherche à répondre aux besoins créés dans le monde professionnel par la "gestion» des populations migrantes sous un angle managérial, comme le souligne l'extrait suivant :

«Aujourd'hui, les questions de diversité et d'interculturalité sont devenues de véritables enjeux pour les entreprises, les organisations et les institutions publiques dans leurs processus de recrutement et de hiérarchisation impactant leurs niveaux de productivité et de rentabilité, leurs relations commerciales ainsi que leur image. Par conséquent, les entreprises, les organisations et les institutions publiques ont besoin d'acquérir de nouvelles compétences basées sur des formations de haut niveau alliant approche analytique permettant de déconstruire de nombreuses notions malmenées par le débat public et gestion pratique de cas concrets donnant l'occasion à chaque participant d'être un acteur de changement au sein de son entreprise/organisation. ${ }^{31}$

La formation est accessible aux titulaires d'un bachelier ou d'une expérience professionnelle évaluée par une commission. Le coût de l'ensemble de la formation varie entre $795 €$ (chômeurs et étudiants), $1395 €$ pour les employeurs et $1095 €$ pour les participants individuels. Cependant, les candidats peuvent choisir certains modules pour $695 €$ chacun.

50 Le certificat offre en 2017 une centaine d'heures de formation en 15 séances (en 2014, 30 séances) articulées autour de trois modules : le premier envisage la migration sous l'angle des circulations et des mobilités, le deuxième porte sur les politiques et les processus d'intégration et le troisième module examine les questions de diversité et de pluralisme. Le programme vaut 10 ECTS et implique la production d'un travail de fin d'études ou d'un «Plan d'action personnel » sur les migrations, la diversité ethnique et les relations interculturelles.

51 La formation est organisée depuis novembre 2014 à la faveur de la mise en œuvre, à la fois, des politiques de diversité et du développement de la mission de «service à la collectivité » des universités qui constitue un moyen d'augmenter leurs sources de financement ${ }^{32}$. Le contenu et le langage utilisés dans les objectifs et le programme semble davantage répondre au souci gestionnaire de la question migratoire; les thématiques choisies reflètent l'approche dominante des migrations ${ }^{33}$ et présente peu d'outils critiques tels qu'ils existent dans la finalité du master en sociologie.

\section{Conclusion}

Les résultats de l'analyse des principaux enseignements sur les migrations internationales dispensés dans le système éducatif belge francophone témoignent à la fois d'un intérêt marginal persistant dans l'enseignement secondaire et de l'émergence de cette thématique dans un cadre professionnalisant dans l'enseignement universitaire.

Dans le secondaire, les enseignements répondent à un besoin de compréhension du monde où la migration est peu perçue comme un phénomène majeur des sociétés contemporaines. C'est pourquoi les autorités éducatives peinent toujours à lui accorder une place plus importante alors que des enseignants témoignent à la fois du vif intérêt des élèves ${ }^{34}$ pour cette matière et de son impact positif sur le climat de la classe dès lors qu'elle leurs fournit des espaces régulés de discussions sur cette thématique et des clés d'analyses qui nuancent les discours politiques et médiatiques hégémoniques. 

plupart, à des personnes qui ont elles-mêmes connu un parcours migratoire ou sont des descendants de migrants. Ceci illustre, comme ce fut le cas pour les études de genre, l'importance du groupe qui porte ces projets/objets et l'existence de luttes pour la reconnaissance des migrations dans le champ hégémonique des sciences sociales, comme le soulignait Abdelmalek Sayad (1991) trente années auparavant.

\section{BIBLIOGRAPHIE}

Beckers, Jacqueline (1998) Les politiques scolaires de l'égalité des chances et de l'égalité des acquis dans l'enseignement secondaire (après 1945), in Grootaers D. (Éd.), Histoire de l'enseignement en Belgique, Bruxelles, Ed du CRISP, pp. 303-371.

Commissariat royal à la politique des immigrés (CRPI) (1990) Pour une cohabitation harmonieuse, Volume III, Données et argumentaires, Bruxelles, mai, 350 p. 
Commission du Dialogue Interculturel (2005) Rapport final et livre des auditions, Bruxelles, mai, $246 \mathrm{p}$.

Cossée Claire ; Miranda Adelina ; Ouali Nouria ; Sehili Djaouida (Éds.) (2012) Le genre au cour des migrations, Paris, Petra Editions, 336 p.

Crispin Jones ; Keith Kimberley (1989) l'Education interculturelle. Concept, contexte et programme, Strasbourg, Edition du Conseil de l'Europe, $217 \mathrm{p}$.

De Coster, Silvain ; Derume, Emile (1962) Retard pédagogique et situation sociale dans le Centre et au Borinage, Bruxelles, Edition de Institut de Sociologie, $117 \mathrm{p}$.

Falaize, Benoit ; Laacher, Smaïn (2014) Posface, in Sayad, A. L'école et les enfants de l'immigration. Essais critiques, Paris, Seuil, pp. 227-236. (la couleur des idées)

Morelli, Anne (1988) L'intérêt d'une histoire des immigrés en Belgique, Cahiers de Clio, automne, $\mathrm{n}^{\circ}$ 95, pp. 61-70.

Morelli, Anne (2004) L'histoire des migrants et l'Histoire, in Ouali N. (Éd.), Trajectoires et dynamiques migratoires de l'immigration marocaine de Belgique, Louvain-La-Neuve, AcademiaBruylant, pp. 13-17.

Morelli, Anne (2011) La lente agonie du Musée de l'Immigration, Revue politique, $n^{\circ}$ 70, Mai-Juin, pp. 48-52.

Ouali, Nouria (2006) Politiques éducatives et immigration : pourquoi avons-nous tant tardé ? in Khader B. et al. (Éds.) Penser l'immigration et l'intégration autrement. Une initiative belge interuniversitaire, Bruxelles, Bruylant, pp. 254-269.

Ouali, Nouria (2007) L'enseignement et les minorités ethniques à Bruxelles, in Delwit P. et al. (Éds.) Bruxelles : ville ouverte. Immigration et diversité culturelle au cœur de l'Europe, Paris, L'Harmattan, pp. 49-68.

Sayad, Abdelmalek (1991) L'immigration ou les paradoxes de l'altérité, Bruxelles, De Boeck-Wesmael, $331 \mathrm{p}$.

Sayad, Abdelmalek (2014) L'école et les enfants de l'immigration. Essais critiques, Paris, Seuil, 236 p. (la couleur des idées).

Vandenberghe, Vincent (1998) L'enseignement en Communauté française de Belgique : un quasimarché, Reflets et Perspectives de la vie économique, XXXVI, 1, pp. 65-75.

Vezinat, Nadège (2016) Sociologie des groupes professionnels, Paris, Armand Colin, 126 p.

\section{NOTES}

1. Entre 1946 et 1974, la Belgique a signé huit conventions bilatérales de main d'œuvre avec l'Italie, la Grèce, l'Espagne, le Maroc, la Turquie, l'Algérie, la Tunisie et la Yougoslavie.

2. Sur la population en âge de travailler (18-60 ans), le monitoring socioéconomique estime la population d' origine étrangère à près de $30 \%$ de la population vivant en Belgique. Si l'on tient compte des moins de 18 ans, la proportion est beaucoup plus importante.

3. Enseignement officiel, enseignement officiel subventionné (communes, provinces et de la région bruxelloise), enseignement libre confessionnel subventionné (catholique), enseignement libre non confessionnel subventionné. Par ailleurs, le secondaire comprend 4 formes d'enseignement : général, technique, artistique et professionnel et est organisé en trois cycles de 
formation de deux ans : le degré d'observation (12-14 ans), le degré d'orientation (14-16 ans) et le degré de détermination (16 à 18 ans).

4. Descendante d'immigrés italiens, elle a édité deux ouvrages sur les migrations : l'histoire des étrangers et de l'immigration en Belgique, de la préhistoire à nos jours, Bruxelles, EVO, 1992. Les émigrants belges : réfugiés de guerre, émigrés économiques, réfugiés politiques ayant quitté nos régions du XVIe siècle à nos jours, Bruxelles, 1998.

5. Communiqué de presse du Collectif «Mémoire Coloniale et Lutte contre les Discriminations » du 29 novembre 2016 invitant les partis politiques à prendre position sur cet enseignement.

6. Du nom de la dernière grande réforme de l'enseignement secondaire mise en place en 1969 qui rompt avec le modèle méritocratique existant et vise à garantir l'égalité des chances et des acquis au plus grand nombre d'élèves. Elle réorganise les six années du secondaire en trois cycles de deux ans (degrés d'observation, d'orientation et de détermination) afin de reculer le moment de l'orientation scolaire définitive et de la fonder sur les intérêts et les aptitudes des élèves identifiées par les enseignants.

7. Le système éducatif repose sur la liberté d'enseignement garantie par la Constitution qui implique la liberté de choix de l'école et celle de créer des écoles. Cette liberté a entraîné une importante diversité d'offres d'enseignement structurées autour de réseaux d'écoles (public, subventionné, libre confessionnel ou pas). Le libre choix de l'école a en outre favorisé la concurrence entre les réseaux et les établissements scolaires entraînant une forte hiérarchisation et ségrégation entre les écoles. Le libre choix des parents, le financement des écoles sur base du nombre d'élèves et la concurrence entre les écoles (incitées à «attirer » leurs clients par la qualité et l'offre éducative) place le système dans un quasi-marché (Vandenberghe, 1998).

8. L'enseignement spécial est réservé aux élèves présentant un handicap physique, mental ou intellectuel ou des difficultés comportementales.

9. Ils sont régulés depuis 1998 par un décret qui interdit les refus pour raison disciplinaire ou pédagogique et oblige les directions à les justifier et à les notifier à l'administration et aux élèves. 10. L'apprentissage de la langue d'origine aux élèves italiens visait à préserver l'unité linguistique de la famille et à faciliter l'insertion scolaire, professionnelle et sociale des jeunes qui retourneraient vivre en Italie. Et l'orientation systématique vers des formations professionnelles était destinée à l'apprentissage d'un «bon » métier (coupe-couture pour les filles, maçon ou mécanicien pour les garçons).

11. Situation socio-économique des parents, taux de chômage et de RMI, taux de délinquance, taux de populations migrantes, etc.

12. Celle-ci supposait en effet de toucher l'ensemble des élèves et des matières par la définition de compétences à transmettre à tous les enfants pour vivre une situation multiculturelle. Il s'agissait de reconnaître et de refléter la dimension multiculturelle des sociétés européennes et de sortir l'enseignement de sa perspective ethnocentrique (Crispin \& Keith 1989). Les responsables éducatifs de la Belgique, comme dans la plupart des États européens, ont interprété ce concept dans son sens le plus étroit, en réservant l'éducation interculturelle aux seuls enfants de migrants comme la directive européenne de 1977 l'exigeait.

13. La contribution en 1985 de Sayad (2014) au rapport de la commission Jacques Berque sur la scolarisation des enfants de l'immigration est particulièrement critique à l'égard de ces enseignements qu'il qualifie de "caricature pédagogique». Il leur reproche notamment de promouvoir l'histoire individuelle et familiale des migrants déconnectée de l'histoire collective et des rapports de domination de classe.

14. L'équivalent de la Commission Stasi en France.

15. Il devait «à la fois présenter l'histoire des immigrations en Belgique, leurs contextes socioéconomiques et politiques, les trajets migratoires collectifs et des récits individuels, et mettre l'accent sur les richesses que représente l'apport des différentes populations étrangères tant au niveau économique que culturel. Sans être un "musée des ethnies» ou des 
« communautés », il devra présenter les apports de ces populations dans «l'art, la cuisine, les métiers, les langages ou le paysage urbain.» (Commission, $2005: 74$ ).

16. En 2001, le gouvernement de la Région de Bruxelles-Capitale inscrivait dans les 12 priorités de son "projet de ville» la création de deux musées: le Musée de l'Europe et le Musée de l'Immigration. Le premier, en place depuis 2006, bénéficie d'énormes moyens financiers (dont ceux de la Commission et du Parlement européens) et occupe le local qui était destiné au musée de l'immigration. En revanche, le musée de l'immigration est délaissé malgré sa défense soutenue par des élus bruxellois issus de l'immigration (Morelli, 2011).

17. Définition du décret officiel de la Communauté flamande de 1998 sur les minorités ethnoculturelles qui subsidie, notamment, l'apprentissage et la transmission de la langue et de la culture d'origine et l'auto-organisation des associations issues de l'immigration. Ce vocable, emprunté à l'administration des Pays-Bas, est désormais utilisé aussi bien dans le langage commun que dans les discours politiques et même dans les travaux scientifiques flamands et francophones.

18. En Belgique, la laïcité est reconnue au même titre que les religions. En conséquence, elle est subsidiée dans les écoles secondaires du réseau public qu'elle organise ou subventionne, au même titre que les religions reconnues (catholique, protestante, orthodoxe, juive et musulmane).

19. Une unité d'acquis d'apprentissage est un ensemble cohérent susceptible d'être évalué selon la discipline.

20. Il existe une multitude d'options de base qui visent l'approfondissement dans toutes les matières.

21. Service général des Affaires pédagogiques, de la Recherche en Pédagogie et du Pilotage de l'Enseignement organisé par la Communauté française, Programme d'études du cours d'histoire $2^{e}$ et $3^{e}$ degrés de l'enseignement général et technique de transition, 50/2000/247.

22. Service général des Affaires pédagogiques, de la Recherche en Pédagogie et du Pilotage de l'Enseignement organisé par la Communauté française, Programme d'études du cours de géographie général et Technique de transition, 57/2000/240.

23. Les autres sont les sciences, sciences économiques, langues, arts, éducation physique qui préfigurent les orientations dans l'enseignement supérieur. Service général des Affaires pédagogiques, de la Recherche en Pédagogie et du Pilotage de l'Enseignement organisé par la Communauté française, Programme d'études du cours de sciences sociales général 2è et 3è degrés, 09/2000/247.

24. Ces cours n'ont pas de programme imposé puisqu'ils sont organisés par les différentes autorités religieuses reconnues. Ils ne font pas l'objet de l'analyse dans cet article.

25. Service général des Affaires pédagogiques, de la Recherche en Pédagogie et du Pilotage de l'Enseignement organisé par la Communauté française, Programme d'études du cours de morale pour les 3 degrés du secondaire, 181/2002/240.

26. Signalons d'emblée qu'aucunes des formations pédagogiques universitaires examinées enseignent les migrations internationales; elles se focalisent essentiellement sur les relations interculturelles, la culture et l'identité.

27. Par exemple, les formations sur l'interculturalité et l'islam (religion, radicalisation) rencontrent un vif succès. Ces formations, certificatives ou pas, coûtent entre 795 euros et 1495 euros (pour 2017-2018) selon le statut de l'apprenant (chômeur ou salarié financé par l'employeur). Comme dans l'enseignement secondaire, la concurrence entre les institutions universitaires les place dans une logique de quasi-marché qui influence le type de formation et leur contenu.

28. Chaque unité d'enseignement (UE) comporte une ou plusieurs activités d'apprentissage (AA).

29. Il est possible que d'autres cours abordent les migrations, mais ils n'ont pu être identifiés à partir de notre procédure de recherche sur les sites universitaires. 
30. C'est un système de congés individuels destiné aux salariés du secteur privé et public autonome pour suivre des formations. A certaines conditions, les personnes gardent leur rémunération.

31. Programme du certificat 2017-2018.

32. Par exemple, dans le contexte de phénomène de "radicalisation des jeunes ", ces deux universités organisent un certificat interuniversitaire de $39 \mathrm{~h}$ sur «Islam et musulmans d'Europe: perspectives historiques et défis contemporains». Il porte sur l'histoire et pensée, politique et religion, religion et société. L'inscription coûte entre $395 €$ et $595 €$.

33. Un de ses objectifs est «Apporter des propositions concrètes à votre organisation en matière de gestion de la diversité ethnoculturelle et de management des relations interculturelles. »

34. Témoignages de plusieurs enseignants que j'ai récoltés en 2015 dans le cadre d'une recherche sur les conditions de travail des jeunes enseignants dans les écoles à Bruxelles. Ces cours ont davantage été développés dans les écoles accueillant des élèves issus des milieux populaires que dans les établissements « élitistes ».

35. Au début des années 1990, par exemple, la politique d'intégration finançait des formations sur les migrations destinées aux corps de police et de gendarmerie, aux pompiers, aux enseignants et aux fonctionnaires publics locaux.

36. Par exemple, l'expertise en droit des étrangers de l'Association de défense des droits des étrangers et celle en pédagogie et en communication interculturelles du Centre bruxellois d'action interculturelle.

37. Le certificat interuniversitaire proposait une session sur « Migration et genre » qui a disparu dans le programme de 2017-2018.

INDEX

Index géographique : France

Mots-clés : enseignement supérieur, éducation, méthodologie

\section{AUTEUR}

\section{NOURIA OUALI}

Professeure associée, Faculté de Philosophie et Sciences sociales de l'Université Libre de Bruxelles; Chercheure au Centre de recherche METICES

nouali@ulb.ac.be 